\title{
Clinical use of TNF revisited: improving penetration of anti-cancer agents by increasing vascular permeability
}

\author{
Ferdy J. Lejeune \\ Multidisciplinary Oncology Center, Lausanne University Hospital, Lausanne, Switzerland \\ J. Clin. Invest. 110:433-435 (2002). doi:10.1172/JCI200216493.
}

A rapid survey of the literature shows that little fundamental progress in cancer chemotherapy has been achieved over the last 20 years. This unfortunate state of affairs may reflect the complexity of the tumor environment and our failure to target both cancer cells and their associated stroma. Although the idea of the cancer cell as a "seed" that can only develop if it is supported by a good "soil" (the stroma with connective tissue and angiogenic vessels) is an old one (see recent retrospective by Fidler [1]), traditional therapies failed to consider that the stroma produces various growth factors that differ from tissue to tissue. Even some of the newer anticancer strategies, which do indeed target the mediators of tumor angiogenesis, still fail to account for the fact that anticancer agents must pass through the soil before they can destroy the seed. Thus, for a long time, clinical oncologists have not been paying enough attention to the fact that poor tumor penetration represents a major impediment to the efficiency of cancer chemotherapeutics. Meanwhile, work in animal models has shown that

\footnotetext{
Address correspondence to: Ferdy J. Lejeune, Multidisciplinary Oncology Centre, Lausanne University Hospital, Level 10, Room 507, Rue du Bugnon 46, 1011 Lausanne, Switzerland. Phone: 41-21-314-01-59;

Fax: 41-21-314-01-67;

E-mail: Ferdy.lejeune@chuv.hospvd.ch. Conflict of interest: No conflict of interest has been declared.

Nonstandard abbreviations used: interstitial fluid pressure (IFP); vascular permeability factor (VPF); endothelial NO synthase (eNOS); peroxynitrite $\left(\mathrm{ONOO}^{-}\right)$; enhanced vascular permeability and retention (EPR); matrix metalloproteinases (MMPs); vascular endothelial cell cadherin (VE-cadherin); isolated limb perfusion (ILP).
}

low permeability of the tumor vasculature and high interstitial fluid pressure (IFP) represent substantial barriers to drug delivery (2).

\section{The dual effects of VEGF}

VEGF, now widely recognized as a major mediator of tumor angiogenesis, was first identified as vascular permeability factor (VPF) (3). Current approaches to blocking angiogenesis include strategies that target VEGF, but little attention has been paid to possible drawbacks, which may include reduced penetration by chemotherapeutic agents administered simultaneously or subsequently. In several animal models, anti-VEGF antibodies or antisense VEGF therapy reduce tumor growth but also consistently reduce intratumoral capillary permeability by as much as 6 -fold, as demonstrated by window chamber or contrast enhanced magnetic resonance imaging (4-6). Vascular permeability changes in response to VEGF result from accumulation of $\mathrm{NO}$, which is produced mainly by endothelial NO synthase (eNOS) (7). Under pathological conditions, including those that prevail in tumors, $\mathrm{NO}$ is converted to peroxynitrite $\left(\mathrm{ONOO}^{-}\right)$, which is responsible for the enhanced vascular permeability and retention (EPR) of drugs in solid tumors. This effect is mediated partly through activation of matrix metalloproteinases (MMPs), which, in turn, generate potent vasoactive mediators such as bradykinin (8). Because inhibition of MMPs has been found to block the induction of EPR in tumors (9), antiVEGF treatment might well restrict the intratumoral penetration of chemotherapy.
Several groups have searched for anti-angiogenic agents free of this inconvenience. For example, neutralizing antibodies to the vascular endothelial cell cadherin (VE-cadherin) may offer an alternative to VEGF that can inhibit tumor angiogenesis without altering vascular permeability (10). In addition, several attempts have been made to selectively augment intratumoral microvascular permeability. Bradykinin selectively increases microvascular permeability in a rat glioma model (8), perhaps by activating the bradykinin B2-receptor, which is upregulated in tumors as compared to normal brain tissue (11).

In spite of the apparent promise of these approaches, the cytokine TNF is the only treatment known that can effect tumor vessel destruction and improve vascular permeability to drugs in a clinical setting (12). TNF occurs as a transmembrane molecule that is made soluble upon activation of a protease. Membrane TNF can induce angiogenesis and, perhaps surprisingly, it can synergize with VEGF to augment vascular permeability. Thus, neutralizing antibodies to TNF abolish the increase of permeability following dual treatment with VEGF and TNF (13). On the other hand, membrane TNF and soluble TNF have proapoptotic properties, especially on endothelium. The cytokine was named after its property to produce hemorrhagic necrosis in experimental tumors, but the clinical application of TNF as systemic treatment was rapidly abandoned because it exerts only a relatively weak antitumor effect and because, as a mediator of septic shock, it is poorly tolerated (reviewed in ref. 12). 
Table 1

Use of TNF in combination to chemotherapy: clinical and preclinical data

\begin{tabular}{|c|c|c|c|c|c|}
\hline Dose & & Effect & Result & Free TNF & Targeted TNF \\
\hline $\begin{array}{l}\text { High } \\
\text { (clinical } \\
[12,14,22])\end{array}$ & $\mathrm{mg}$ & $\begin{array}{l}\text { Deactivation of } \alpha_{v} \beta_{3} \text { integrin } \\
\text { in endothelial cells (17) }\end{array}$ & $\begin{array}{l}\text { Destruction of } \\
\text { angiogenic vessels }\end{array}$ & $\begin{array}{l}\text { Administered in isolated } \\
\text { limb perfusion }\end{array}$ & \\
\hline $\begin{array}{l}\text { Low } \\
\text { (preclinical only } \\
{[15,20] \text { ) }}\end{array}$ & ng & $\begin{array}{l}\text { Increased intratumoral } \\
\text { vessel permeability }\end{array}$ & $\begin{array}{l}\text { Increased drug penetration } \\
\text { in tumor }(15,20,21)\end{array}$ & Systemic administration & \\
\hline $\begin{array}{l}\text { Very low } \\
\text { (preclinical } \\
\text { only [21]) }\end{array}$ & pg & & $\begin{array}{l}\text { Increased drug penetration } \\
\text { in tumor }\end{array}$ & & $\begin{array}{l}\text { Systemic } \\
\text { administration }\end{array}$ \\
\hline
\end{tabular}

\section{TNF in the cancer clinic}

In 1988, we had the idea of using high-dose TNF in isolated limb perfusion (ILP) in combination to chemotherapy for locally advanced melanomas and sarcomas of the limbs. This treatment, we found, yields a very high complete response rate (14). Angiographic and histologic studies revealed that its effect was due to selective destruction of the tumor associated vessels and that vessels in normal tissues were spared. These early clinical results inspired experimental studies, in a bedside-tobench transfer, and it emerged that either systemic or intratumoral application of TNF can increase tumor microvasculature flow and permeability, and hence penetration of antibodies in a mouse colon cancer xenograph model (15). TNF also reduces IFP, as evidenced by an increase in capillary filtration with no effect on capillary tone (16). Taken together, these results indicate a selective effect of TNF on angiogenic endothelial cells.

An explanation for this remarkable selectivity for growing but not quiescent vascular tissues came from in vitro and in vivo studies on endothelial biology. TNF, in combination with IFN- $\gamma$, deactivates the integrin $\alpha_{V} \beta_{3}$, which is only expressed by angiogenic endothelial cells. Since this integrin is essential for proliferation and survival, treated angiogenic ECs fail to adhere to ECM proteins in the microvessels, and they undergo massive apoptosis (17). In a rat limb soft tissue sarcoma model, it was found that perfusion with TNF selectively enhances melphalan penetration into tumors but not in muscle tissue (18). Doxorubicin penetration of soft tissue sarcomas and osteosarcomas is also improved by this treatment (19). We, as well as others (15, 20), have proposed that TNF exerts two distinct effects that are selective for angiogenic vessels, namely an early increase of tumor vessels permeability, which can be induced with a low dose of TNF, and a later increase in vascular apoptosis, which appears to require high doses.

\section{Prospects for targeted delivery of TNF}

In this issue of the JCI, Curnis et al. (21) demonstrate that extremely low doses of TNF are sufficient to increase the penetration of doxorubicin and melphalan into melanoma and lymphoma models when the cytokine is coupled with a peptide that targets it specifically to the endothelial cell. The GNGRC peptide is a ligand of aminopeptidase $\mathrm{N}$ (CD13) and readily binds to endothelial cells. The authors show that dual treatment with chemotherapeutic agents and a TNF-GNGRC peptide fusion can be curative in established lymphoma transplants. Importantly, they confirm that the latter agent is not toxic, and they also determine that its effects on vascular permeability are mediated by TNF-R1 (p55) - a receptor that has been shown to be required for intracellular signaling leading to either NFKB activation or apoptosis. Interestingly, a low dose of the compound is more effective than a medium dose because it does not induce the production of soluble TNF receptors, which would neutralize TNF. Thus, as shown in Table 1, picogram level beneficial effects of this modified TNF must be considered alongside the previously established nanogram level effects of native TNF on vascular permeability and the milligram level effects on vascular cell survival.

Our earlier ILP results in melanoma and sarcoma showed that TNF can increase the therapeutic efficiency of chemotherapeutic agents, even those of marginal activity. For example, melphalan, a drug with no activity in soft tissue sarcoma, can produce $30 \%$ complete responses in large sarcomas of the limbs when administered with TNF (22). Likewise, in a rat sarcoma model, systemic administration of low-dose TNF augments the antitumor activity of a liposomal formulation of doxorubicin (20). Thus, although it was abandoned ten years ago by medical oncologists because of its systemic toxicity, TNF may be due for a comeback in the clinic as a systemic treatment that can prepare the soil so that chemotherapy can work at optimal efficiency on the seed of tumor survival and growth.

1. Fidler, I.J. 2001. Seed and soil revisited: contribution of the organ microenvironment to cancer metastasis. Surg. Oncol. Clin. N. Am. 10:257-269, vii-viiii.

2. Jain, R.K. 1999. Understanding barriers to drug delivery: high resolution in vivo imaging is key. Clin. Cancer. Res. 5:1605-1606.

3. Senger, D.R., et al. 1993. Vascular permeability factor (VPF, VEGF) in tumor biology. Cancer. Metastasis. Rev. 12:303-324.

4. Lichtenbeld, H.C., Ferarra, N., Jain, R.K., and Munn, L.L. 1999. Effect of local anti-VEGF antibody treatment on tumor microvessel permeability. Microvasc. Res. 57:357-362.

5. Oku, T., et al. 1998. Tumor growth modulation by sense and antisense vascular endothelial growth factor gene expression: effects on angiogenesis, vascular permeability, blood volume, blood flow, fluorodeoxyglucose uptake, and proliferation of human melanoma intracerebral xenografts. Cancer. Res. 58:4185-4192.

6. Pham, C.D., et al. 1998. Magnetic resonance imaging detects suppression of tumor vascular permeability after administration of antibody to 
vascular endothelial growth factor. Cancer. Invest. 16:225-230.

7. Fukumura, D., et al. 2001. Predominant role of endothelial nitric oxide synthase in vascular endothelial growth factor-induced angiogenesis and vascular permeability. Proc. Natl. Acad. Sci. USA. 98:2604-2609.

8. Nakano, S., Matsukado, K., and Black, K.L. 1996. Increased brain tumor microvessel permeability after intracarotid bradykinin infusion is mediated by nitric oxide. Cancer. Res. 56:4027-4031.

9. Wu, J., et al. 2001. Enhanced vascular permeability in solid tumor involving peroxynitrite and matrix metalloproteinases. Jpn. J. Cancer. Res. 92:439-451.

10. Liao, F., et al. 2002. Selective targeting of angiogenic tumor vasculature by vascular endothelialcadherin antibody inhibits tumor growth without affecting vascular permeability. Cancer. Res. 62:2567-2575.

11. Matsukado, K., Sugita, M., and Black, K.L. 1998. Intracarotid low dose bradykinin infusion selectively increases tumor permeability through activation of bradykinin $\mathrm{B} 2$ receptors in malignant gliomas. Brain. Res. 792:10-15.
12. Lejeune, F.J., Ruegg, C., and Lienard, D. 1998 Clinical applications of TNF-alpha in cancer. Curr. Opin. Immunol. 10:573-580.

13. Clauss, M., et al. 2001. A permissive role for tumor necrosis factor in vascular endothelia growth factor-induced vascular permeability. Blood. 97:1321-1329.

14. Lienard, D., Ewalenko, P., Delmotte, J.J., Renard, N., and Lejeune, F.J. 1992. High-dose recombinant tumor necrosis factor alpha in combination with interferon gamma and melphalan in isola tion perfusion of the limbs for melanoma and sarcoma. J. Clin. Oncol. 10:52-60.

15. Folli, S, et al. 1993. Tumor-necrosis factor can enhance radio-antibody uptake in human colon carcinoma xenografts by increasing vascular permeability. Int. J. Cancer. 53:829-836.

16. Jahr, J., and Grande, P.O. 1996. In vivo effects of tumor necrosis factor-alpha on capillary permeability and vascular tone in a skeletal muscle. Acta. Anaesthesiol. Scand. 40:256-261.

17. Ruegg, C., et al. 1998. Evidence for the involvement of endothelial cell integrin alphaVbeta3 in the disruption of the tumor vasculature induced by TNF and IFN-gamma. Nat. Med. 4:408-414.
18. de Wilt, J.H., et al. 2000. Tumour necrosis factor alpha increases melphalan concentration in tumour tissue after isolated limb perfusion. $B r . J$. Cancer. 82:1000-1003.

19. van der Veen, A.H., et al. 2000. TNF-alpha augments intratumoural concentrations of doxorubicin in TNF- alpha-based isolated limb perfusion in rat sarcoma models and enhances anti-tumour effects. Br. J. Cancer. 82:973-980.

20. Ten Hagen, T.L., et al. 2000. Low-dose tumor necrosis factor-alpha augments antitumor activity of stealth liposomal doxorubicin (DOXIL) in soft tissue sarcoma-bearing rats. Int. J. Cancer. 87:829-837.

21. Curnis, F., Sacchi, A., and Corti, A. 2002 Improving chemotherapeutic drug penetration in tumors by vascular targeting and barrier alteration. J. Clin. Invest. 110:475-482. doi:10.1172/JCI200215223.

22. Eggermont, A.M., et al. 1996. Isolated limb perfusion with high-dose tumor necrosis factoralpha in combination with interferon-gamma and melphalan for nonresectable extremity soft tissue sarcomas: a multicenter trial. J. Clin. Oncol. 14:2653-2665 